\title{
The European Photon Imaging Camera on XMM-Newton: The pn-CCD camera
}

L. Strüder ${ }^{1}$, U. Briel ${ }^{1}$, K. Dennerl ${ }^{1}$, R. Hartmann ${ }^{2}$, E. Kendziorra ${ }^{4}$, N. Meidinger ${ }^{1}$, E. Pfeffermann $^{1}$, C. Reppin ${ }^{1}$, B. Aschenbach ${ }^{1}$, W. Bornemann ${ }^{1}$, H. Bräuninger ${ }^{1}$, W. Burkert ${ }^{1}$, M. Elender ${ }^{1}$, M. Freyberg ${ }^{1}$, F. Haberl ${ }^{1}$, G. Hartner ${ }^{1}$, F. Heuschmann ${ }^{1}$, H. Hippmann ${ }^{1}$, E. Kastelic ${ }^{1}$, S. Kemmer ${ }^{1}$, G. Kettenring ${ }^{1}$, W. Kink ${ }^{1}$, N. Krause ${ }^{1}$, S. Müller ${ }^{1}$, A. Oppitz ${ }^{1}$, W. Pietsch ${ }^{1}$, M. Popp ${ }^{1}$, P. Predehl ${ }^{1}$, A. Read ${ }^{1}$, K. H. Stephan ${ }^{1}$, D. Stötter ${ }^{1}$, J. Trümper ${ }^{1}$, P. Holl ${ }^{2}$, J. Kemmer ${ }^{2}$, H. Soltau ${ }^{2}$, R. Stötter ${ }^{2}$, U. Weber ${ }^{2}$, U. Weichert ${ }^{2}$, C. von Zanthier ${ }^{2}$, D. Carathanassis ${ }^{3}$, G. Lutz $^{3}$, R. H. Richter ${ }^{3}$, P. Solc ${ }^{3}$, H. Böttcher ${ }^{4}$, M. Kuster ${ }^{4}$, R. Staubert ${ }^{4}$, A. Abbey ${ }^{5}$, A. Holland ${ }^{5}$, M. Turner ${ }^{5}$, M. Balasini ${ }^{6}$, G. F. Bignami ${ }^{6}$,

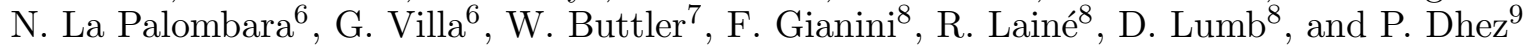

1 Max-Planck-Institut für extraterrestrische Physik, Giessenbachstraße, 85748 Garching, Germany

${ }^{2}$ KETEK GmbH, Am Isarbach 30, 85764 Oberschleißheim, Germany

3 Max-Planck-Institut für Physik, Föhringer Ring 6, 80805 München, Germany

${ }^{4}$ Institut für Astronomie und Astrophysik, Waldhäuser Str. 64, 72076 Tübingen, Germany

5 X-ray Astronomy Group, Dept. of Physics and Astronomy, Leicester University, Leicester, LE1 7RH, UK

${ }^{6}$ Istituto di Fisica Cosmica "G. Occhialini", CNR, Via E. Bassini 15/A, 20133 Milano, Italy

7 Ingenieurbüro Buttler, Eschenburg 55, 45276 Essen, Germany

8 ESTEC, PX, Postbus 299, 2200 AG Noordwijk, The Netherlands

${ }^{9}$ LURE, Bât. 209 D, Université Paris Sud, 91405 Orsay, France

Received 2 October 2000 / Accepted 27 October 2000

\begin{abstract}
The European Photon Imaging Camera (EPIC) consortium has provided the focal plane instruments for the three X-ray mirror systems on XMM-Newton. Two cameras with a reflecting grating spectrometer in the optical path are equipped with MOS type CCDs as focal plane detectors (Turner 2001), the telescope with the full photon flux operates the novel pn-CCD as an imaging X-ray spectrometer. The pn-CCD camera system was developed under the leadership of the Max-Planck-Institut für extraterrestrische Physik (MPE), Garching. The concept of the pn-CCD is described as well as the different operational modes of the camera system. The electrical, mechanical and thermal design of the focal plane and camera is briefly treated. The in-orbit performance is described in terms of energy resolution, quantum efficiency, time resolution, long term stability and charged particle background. Special emphasis is given to the radiation hardening of the devices and the measured and expected degradation due to radiation damage of ionizing particles in the first 9 months of in orbit operation.
\end{abstract}

Key words. XMM-Newton - back illuminated pn-CCDs - radiation hardness - energy resolution - quantum efficiency - particle and flourescence background

\section{Introduction}

For ESA's X-ray Multi Mirror (XMM) mission, we have developed a $6 \times 6 \mathrm{~cm}^{2}$ monolithic X-ray CCD array (Strüder et al. 1990) with high detection efficiency up to $15 \mathrm{keV}$, low noise level $\left(\mathrm{ENC} \approx 5 \mathrm{e}^{-}\right)$and ultrafast readout. The detector was tailored to the requirements of the XMM telescope performance, concerning angular

Send offprint requests to: L. Strüder,

e-mail: lts@hll.mpg.de

* Based on observations with XMM-Newton, an ESA Science Mission with instruments and contributions directly funded by ESA Member States and the USA (NASA). resolution, collecting area, energy bandwidth and field of view (FoV).

Conceptually the pn-CCD, the heart of the MPE focal plane detector, is a derivative of the silicon drift detector proposed in 1983 by Gatti \& Rehak (1984). In the following years the basic concept was modified, simulated and designed in detail by Strüder et al. (1987). N-channel JFET electronics was integrated in 1992 (Pinotti et al. $1993)$ and the first reasonably working devices were produced in 1993.

The flight type large area detectors were fabricated in 1997 in the MPI semiconductor laboratory, with a sufficiently high yield to equip XMM and ABRIXAS, 
a German X-ray satellite, with defect free focal plane pnCCDs (Strüder et al. 1997; Soltau et al. 2000).

The in-orbit commissioning of XMM's scientific payload was completed in the middle of March 2000 - three months after launch; calibration and performance verification terminated in July. Since then, the official observing programme is under way. This contribution summarizes the basic instrument features as previously planned and implemented in orbit to date, as well as their scientifically relevant measured performance in space. We have included orbit data up to September 2000.

\section{The concept of fully depleted pn-CCDs}

The principle of sideward depletion in high resistivity silicon is the basis of a large variety of novel silicon detectors, such as silicon drift detectors, controlled drift detectors, active pixel sensors - and pn-CCDs.

\subsection{The camera concept}

The angular resolution of the XMM X-ray telescope in front of the pn-CCD camera (mirror flight model 2, FM2) is 15 arcsec half energy width (HEW) at $1.5 \mathrm{keV}$ and $8 \mathrm{keV}$. This translates to $540 \mu \mathrm{m}$ position resolution required in the focal plane. For a given telescope performance the concept of sideward depletion allows for an optimum adaption of the pixel size to the X-ray optics, varying from $30 \mu \mathrm{m}$ up to $300 \mu \mathrm{m}$. The FWHM of the point spread function (PSF) is 6.6 arcsec. A pixel size of $150 \mu \mathrm{m} \times 150 \mu \mathrm{m}(4.1$ arcsec $)$ was chosen, with a position resolution of $120 \mu \mathrm{m}$, resulting in an equivalent angular resolving capability of 3.3 arcsec. The energy response is higher than $90 \%$ at $10 \mathrm{keV}$ because of the sensitive thickness of $300 \mu \mathrm{m}$. The low-energy response is given by the very shallow implant of the $\mathrm{p}^{+}$back contact; the effective "dead" layer is of the order of $300 \AA$ (Hartmann et al. 1997). High time resolution is a consequence of the parallel readout of 64 channels per subunit; in total 768 channels for the entire camera. High radiation hardness is built in by avoiding active MOS structures and by the fast transfer of the charge in a depth of more than $10 \mu \mathrm{m}$ below the surface. For low energy protons, imaged through the X-ray optics (Aschenbach 2001) the pn-CCD is "self shielding", because the ionizing radiation has to propagate through $290 \mu \mathrm{m}$ of silicon before damaging the transfer channel and decreasing charge transfer efficiency (CTE). As there is only a negligible transmission of protons through the $\mathrm{X}$-ray optics above $500 \mathrm{keV}$, there is no problem for the pn-CCD with low energy protons at all. Measurements in a proton accelerator by Meidinger et al. (2000) with up to $210^{9} 10 \mathrm{MeV}$ protons per $\mathrm{cm}^{2}$, equivalent to 4 times the expected 10 year XMM irradiation in space, only showed a degradation of about $30 \mathrm{eV}$ in the FWHM of the $\mathrm{MnK}_{\alpha}$ line. Kendziorra et al. (2000) tested the pn-CCDs with low energy proton up to $1.410^{9}$ protons per $\mathrm{cm}^{2}$. No change of the detector's properties was seen. This proton irradiation at energies between $1 \mathrm{keV}$ and $300 \mathrm{keV}$ with prominent

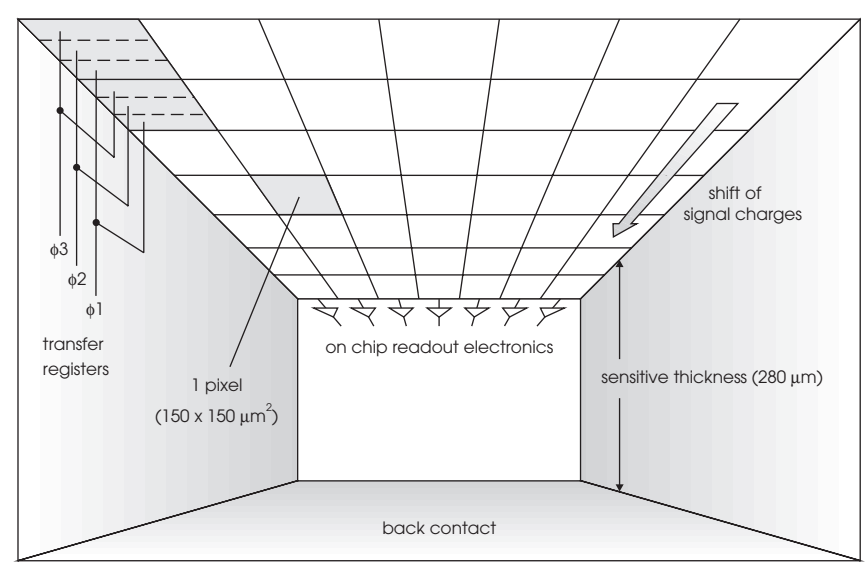

Fig. 1. Inside the pn-CCD. The X-rays hit the device from the backside (bottom). The charges are collected in the electron potential minimum $10 \mu \mathrm{m}$ from the surface having the pixel structure. After integration, they are transferred to the onchip amplifier. Each CCD column is terminated by an on-chip JFET amplifier

peaks at $70 \mathrm{keV}$ and $170 \mathrm{keV}$, was a factor of 1000 above the expected low energy proton flux in orbit. Up to now, no measurable degradation due to radiation damage was found in orbit.

\subsection{The basic principles of $p n-C C D s$}

The schematic view into the pn-CCD in Fig. 1 already introduces intuitively the advantages of the concept: X-rays hit the detector from the rear side. In case of an X-ray interaction with the silicon atoms, electrons and holes are generated. The average energy required to form an electron-hole pair is $3.7 \mathrm{eV}$ at $-90{ }^{\circ} \mathrm{C}$. The strong electric fields in the pn-CCD detector separate the electrons and holes before they recombine. Signal charges (in our case electrons), are drifted to the potential minimum and stored under the transfer registers. The positively charged holes move to the negatively biased back side, where they are "absorbed". The electrons, captured in the potential wells $10 \mu \mathrm{m}$ below the surface can be tansferred towards the readout nodes upon command, conserving the local charge distribution patterns from the ionization process. As can be seen in Fig. 1, each CCD line is terminated by a readout amplifier. The focal plane layout is depicted in Fig. 2. Four individual quadrants each having 3 pn-CCD subunits with a format of $200 \times 64$ pixel are operated in parallel.

The spatially uniform detector quality over the entire field of view is realized by the monolithic fabrication of 12 individually operated $3 \times 1 \mathrm{~cm}^{2}$ pn-CCDs on a single wafer (see Fig. 2). No inhomogenities were observed in the tested energy range from $700 \mathrm{eV}$ up to $8 \mathrm{keV}$, the measured flatness of the homogeneity measurements was always limited by Poisson statistics. Figure 2 shows the insensitive or partially sensitive gaps in between the different CCDs and quadrants. As all CCDs are monolithically integrated 
Table 1. Parameters of the six standard readout modes as implemented in-orbit. The energy flux in the last column* was derived in the energy band from $0.1 \mathrm{keV}$ to $10 \mathrm{keV}$ with the assumption of an unabsorbed power law with photon index 2.0 , observed with the thin filter

\begin{tabular}{|c|c|c|c|c|c|}
\hline mode & $\begin{array}{l}\text { field of view (FoV) } \\
\text { in pixel format } \\
\text { in arcmin }\end{array}$ & $\begin{array}{l}\text { time resolution } \\
\text { resolution } \\
\text { in } \mathrm{ms}\end{array}$ & $\begin{array}{c}\text { out of time } \\
\text { (OOT) events } \\
\text { in } \%\end{array}$ & $\begin{array}{c}\text { life time } \\
\text { with OOT events } \\
\text { in } \%\end{array}$ & $\begin{array}{c}\text { brightest point source } \\
\text { for XMM } \\
\text { in counts per sec } \\
\text { in erg cm } \mathrm{cm}^{-2} \mathrm{~s}^{-1} * \\
\end{array}$ \\
\hline $\begin{array}{l}\text { full frame } \\
\text { (1) }\end{array}$ & $\begin{array}{c}398 \times 384 \\
27.2 \times 26.2\end{array}$ & 73.3 & $\overline{6.2}$ & $\overline{999.9}$ & $\begin{array}{c}6 \\
8.110^{-12}\end{array}$ \\
\hline $\begin{array}{l}\text { extended full frame } \\
(2)\end{array}$ & $\begin{array}{c}398 \times 384 \\
27.2 \times 26.2 \\
\end{array}$ & 199.2 & 2.3 & 100 & $\begin{array}{c}\text { for extended sources } \\
\text { only }\end{array}$ \\
\hline $\begin{array}{c}\text { large window } \\
(3)\end{array}$ & $\begin{array}{c}198 \times 384 \\
13.5 \times 26.2\end{array}$ & 47.7 & 0.15 & 94.9 & $\begin{array}{c}9 \\
1.210^{-11}\end{array}$ \\
\hline $\begin{array}{l}\text { small window } \\
\text { (4) }\end{array}$ & $\begin{array}{c}63 \times 64 \\
4.3 \times 4.4\end{array}$ & 5.7 & 1.1 & 71.0 & $\begin{array}{c}104 \\
1.410^{-10}\end{array}$ \\
\hline $\begin{array}{l}\text { timing } \\
(5)\end{array}$ & $\begin{array}{c}199 \times 64 \\
13.6 \times 4.4\end{array}$ & 0.03 & 100 & 99.5 & $\begin{array}{c}4000 \\
5.910^{-9}\end{array}$ \\
\hline $\begin{array}{c}\text { burst } \\
(6)\end{array}$ & $\begin{array}{c}20 \times 64 \\
1.4 \times 4.4\end{array}$ & 0.007 & $\begin{array}{l}\text { depends on } \\
\text { PSF }\end{array}$ & 3.0 & $\begin{array}{c}60000 \\
8.110^{-8}\end{array}$ \\
\hline
\end{tabular}

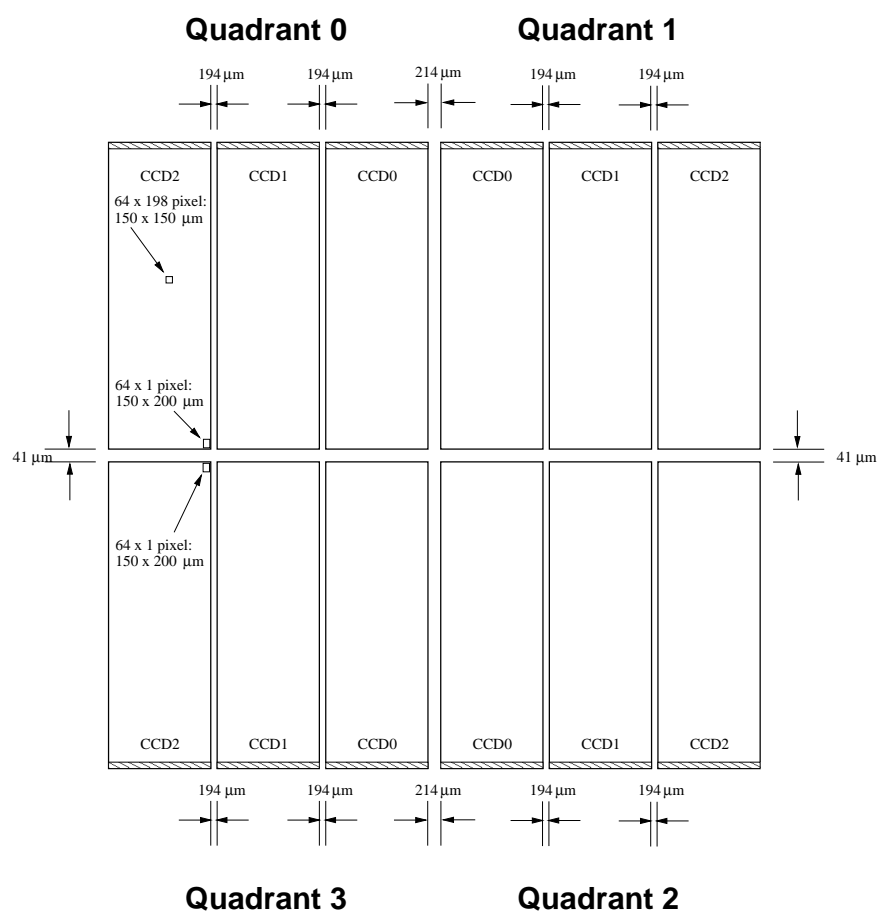

Fig. 2. Overview of the internal boundaries of the pn-CCD focal plane. The division of the focal plane in subunits was made because of redundancy reasons. The focal point of the X-ray telescope is in CCD0, quadrant 1 . About $97 \%$ of the telescopes field of view is covered by the focal plane. About $6 \mathrm{~cm}^{2}$ of the CCD's sensitive area are outside the field of view and is used for background studies. The world's largest X-ray CCD with a sensitive area of $36 \mathrm{~cm}^{2}$ was fabricated in the MPIsemiconductor laboratory

on a single 4 inch wafer, the relative adjustment of the chips, i.e. all pixels, is known with a precision of better than $1 \mu \mathrm{m}$.

The improvement of the energy resolution of the detector requires cooling, to suppress the thermally

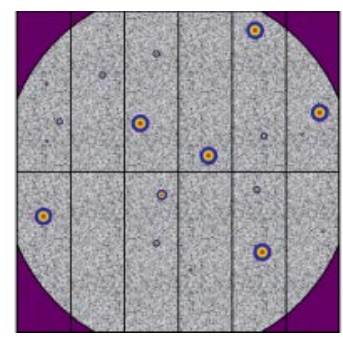

Full Frame \& Extended Full Frame

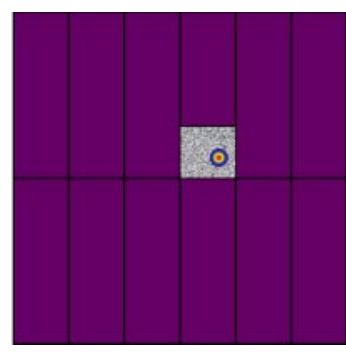

Small Window in Quadrant 1

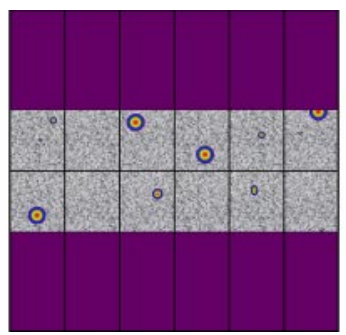

Large Window

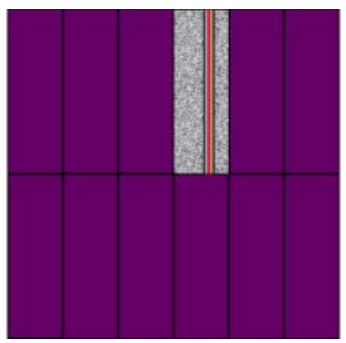

Timing Mode in Quadrant 1
Fig. 3. Operating modes of the pn-CCD camera a) Full frame and extended full frame mode, b) Large window mode, $\mathbf{c}$ ) small window mode and $\mathbf{d}$ ) timing mode and burst mode

generated leakage current. We have chosen a temperature of $-90^{\circ} \mathrm{C}$, reducing the leakage current to less than $0.1 \mathrm{e}^{-}$ per pixel and per readout cycle of $73 \mathrm{~ms}$. Taking into account the residual partial pressure inside the camera of less than $10^{-5}$ mbar, formation of monolayers of e.g. ice on the radiation entrance window should not occur.

\subsection{Operating modes}

CCDs have originally been designed for photon intensity imaging, not single photon counting in a spectroscopic mode. To make CCDs useful for X-ray imaging and spectroscopic applications simultaneously, they must be operated such that only one X-ray photon hits the 
detector without an overlap in time and position of another photon. The design of the readout modes was driven by the assumption that the local photon flux should be below $\frac{1}{40}$ events per pixel and integration time. To adapt the X-ray camera readout mode to the point source brightness, the integration time of the CCD camera can be shortened - by reducing the area to be read out. Under the cost of sensitive area the photon flux can be increased. Figure 3 shows which part of the CCD array is read out in the different modes. Table 1 contains the most important parameters of the pn-CCD readout modes. A detailed summary was given by Kuster et al. (1999).

\subsubsection{The full frame and the extended full frame mode}

In the pn-CCD's full frame mode, a complete readout cycle takes $73.3 \mathrm{~ms}$ for one individual CCD subunit. Within that time, $4.6 \mathrm{~ms}(200 \times 23 \mu \mathrm{s})$ are needed for the readout itself, and $68.7 \mathrm{~ms}$ are used for the integration of the image. This timing schedule leads to $6.2 \%$ "out-of-time events", i.e. events which hit the detector during the readout. In the extended full frame mode the X-ray integration time is $199.2 \mathrm{~ms}$ with again $4.6 \mathrm{~ms}$ readout, leading to $2.3 \%$ of out-of-time events only. The extended full frame mode is suggested for the observation of extended objects. The time resolution in both cases is the total cycle time, i.e. photon integration time plus readout time.

\subsubsection{The large and small window mode}

Both window modes are operated similar to a conventional frame store mode, where in our case the storage area is not covered by an X-ray blocking shielding. No bright source should be focussed on the storage area, because it could contaminate the information integrated in the image area.

The window modes as shown in Fig. 3 reduce the field of view to reduce the number of out-of-time events and to improve the time resolution and finally increase the pileup limit for bright sources. In the large window mode the inner half of the CCD is used for imaging, then rapidly transferred towards the readout node (720 ns per CCD line) and eventually read out, similar to the full frame mode. During the fast transfer the image outside the defined FoV is automatically cleared. The time resolution is lowered to $47.7 \mathrm{~ms}$ and the amount of out-of-time events drop below $0.2 \%$ in the large window mode. The small window mode operates comparably. The difference is that the field of view is further reduced to $63 \times 64$ pixels and only one quadrant is operated instead of four. The time resolution drops to $5.7 \mathrm{~ms}$ and out-of-time event contribution is $1.1 \%$. The small window mode only uses CCD0 of quadrant 1 , i.e. the focal CCD.

\subsubsection{The timing and burst mode}

Only the CCD0 from quadrant 1 is operated in the fast modes. The timing mode forms macro-pixels of

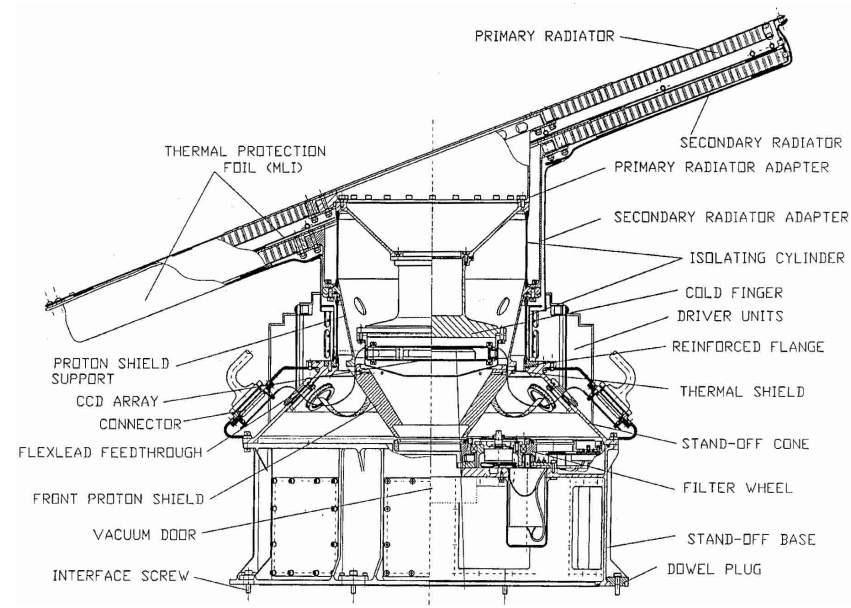

Fig. 4. Mechanical structure of the pn-CCD camera system. $\mathrm{X}$-rays imaged through the telescope enter the detector from the bottom

$10 \times 1$ pixels. In one dimension (64) the position resolution is maintained while the other 10 pixels are read out only after 9 fast transfers without electronic processing. That means, that 10 pixels along a column are integrated on the readout node. The position information within those 10 pixels is lost, it is conserved in the perpendicular direction. The time resolution is then $30 \mu \mathrm{s}$.

The burst mode rapidly transfers 179 pixels and then reads the content of CCD0 in the conventional way. This allows for a $7 \mu$ s time resolution and up to 60000 counts per second in the PSF. After each read out, the entire CCD is cleared from signals. The duty cycle (life time) in this mode is only $3 \%$. The strongest sources can be observed in that mode.

\subsection{The mechanical and thermal concept of the pn-CCD camera system}

The camera housing is mainly made out of aluminum, the average integrated equivalent aluminum thickness, shielding the CCD from cosmic ionizing radiation is roughly $3 \mathrm{~cm}$. The aluminum (AlZnMgCu1,5) contains $\mathrm{Si}, \mathrm{Fe}$, $\mathrm{Cu}, \mathrm{Mn}, \mathrm{Mg}, \mathrm{Cr}, \mathrm{Zn}$ and Ti. In total, these "trace" elements represent about $10 \%$ of the total mass. Figure 4 shows a cross section through the pn-CCD camera system. The main components are the radiator, cold finger, proton shield and the printed circuit board with the integrated preamplifiers (CMX and TMX) and the pn-CCD, mounted in an invar ring. The interconnections between the CCD and the surrounding electronics on the PC board are wedge-bonded. About 900 bonds were required, all individually coated, to improve their mechanical stability.

The invar consists mainly of $\mathrm{Ni}, \mathrm{Mn}, \mathrm{Si}, \mathrm{C}$ and $\mathrm{Fe}$. The PC board contains beside its Mo core $\mathrm{Cu}$ lines as metallization layer ${ }^{1}$. A spider type support structure,

\footnotetext{
1 As you will see later, most of the $K_{\alpha}$ and $K_{\beta}$ lines of the mentioned atoms appear as fluorescence lines in the calibration spectra in Fig. 6.
} 
Table 2. Filter properties $(\mathrm{Sn}=\mathrm{Tin}, \mathrm{PP}=$ Polypropylene, $\mathrm{PI}=$ Polyimide, $\mathrm{Al}=$ Aluminium)

\begin{tabular}{lcccc}
\hline filter & $\begin{array}{c}\text { layer 1 } \\
\mu \mathrm{g} \mathrm{cm}^{-2}\end{array}$ & $\begin{array}{c}\text { layer 2 } \\
\mu \mathrm{g} \mathrm{cm}^{-2}\end{array}$ & $\begin{array}{c}\text { layer 3 } \\
\mu \mathrm{g} \mathrm{cm}^{-2}\end{array}$ & $\begin{array}{c}\text { layer } 4 \\
\mu \mathrm{g} \mathrm{cm}^{-2}\end{array}$ \\
\hline \hline open & - & - & - & - \\
position 1 & & & & - \\
\hline closed & $\mathrm{Al}$ & - & - & - \\
position 2 & 270200 & - & - & - \\
\hline $2 \times$ thin & $\mathrm{Al}$ & $\mathrm{PI}$ & - & - \\
position 3, 4 & 10.8 & 22.4 & - & - \\
\hline medium & $\mathrm{Al}$ & $\mathrm{PI}$ & - & - \\
position 5 & 21.6 & 22.4 & - & - \\
\hline thick & $\mathrm{Sn}$ & $\mathrm{Al}$ & $\mathrm{PP}$ & $\mathrm{Al}$ \\
position 6 & 18 & 28 & 27.5 & 28 \\
\hline
\end{tabular}

smoothly pressed onto the PC board and on the CCD wafer act as a mechanical stabilization of the main components of the camera head.

Between stand-off cone and stand-off base a filter wheel is implemented with 6 filter positions: Four positions carry filters of different thicknesses (see Table 2), one position is open and the closed position is realized by a $1 \mathrm{~mm}$ thick aluminum plate, to block ionizing radiation imaged through the mirror system. Four positions are equipped with thick, medium and two thin filters as specified in Table 2. A calibration fluorescent source $\left(\mathrm{AlK}_{\alpha}\right.$ and $\mathrm{MnK}_{\alpha}$ and $\mathrm{MnK}_{\beta}, 1.487 \mathrm{keV}$ and $5.894 \mathrm{keV}$ and $6.489 \mathrm{keV}$ resp.) can illuminate the CCD through the filter wheel upon command. The total count rate of the calibration source on the detector is of the order of 100 counts per second, the half-life of ${ }^{55} \mathrm{Fe}$ is 2.7 years. The spatial distribution of the X-rays from the calibration source is inhomogeneous over the field of view.

The actual planning foresees an operating temperature of the pn-CCD of $-90{ }^{\circ} \mathrm{C}$ during the whole mission. An active temperature control stabilizes the chip temperature to better than $0.1 \mathrm{~K}$. The temperature is measured on the CCD directly and on the invar ring. The thermal design was made to achieve CCD temperatures as low as $-140{ }^{\circ} \mathrm{C}$ while dissipating $0.9 \mathrm{~W}$ of power in the focal plane.

The electronic concept was designed with a high degree of redundancy. The four quadrants of the CCD wafer are operated and controlled seperately. In addition the three CCDs of one quadrant can be electrically adjusted almost independently. The relevant supply voltages and currents of each CCD are programmable from ground. This enables the instrument team to modify operating conditions in case of performance degradation, if needed.

\section{Instrument performance}

The pn-CCD camera system was submitted to an intensive ground calibration programme. A detector response matrix was filled with measured data and modelled interpolations, where no data were available. Within the measurement accuracy the in-flight performance does not

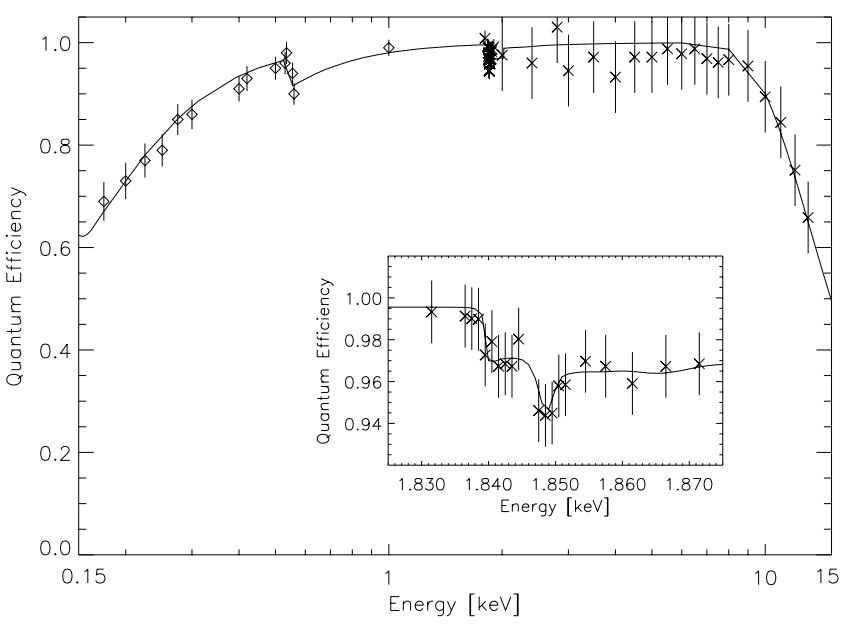

Fig. 5. Quantum efficiency (QE) of the pn-CCD with a fully depleted thickness of $300 \mu \mathrm{m}$

deviate from on-ground calibrations under the same operating conditions (Briel et al. 2000).

\subsection{Quantum efficiency}

The fully depleted $300 \mu \mathrm{m}$ of silicon determines the detection efficiency on the high energy end, while the quality of the radiation entrance window is responsible for the low energy response. Figure 5 shows the result of the absolute quantum efficiency calibration at PTB (BESSY synchrotron in Berlin) and LURE (synchrotron in Orsay, Paris). All measurements were made under conditions comparable to space operation. The drop of quantum efficiency (QE) at the lowest energies is caused by the properties of the silicon L-edge. The absorption length of X-rays in silicon at e.g. $150 \mathrm{eV}$ is only $30 \mathrm{~nm}$. A thin oxide layer of the order of $20 \mathrm{~nm}$ already absorbs about one half of the incident photons. The drop of about $5 \%$ of $\mathrm{QE}$ at $528 \mathrm{eV}$ is due to the additional absorption in the $\mathrm{SiO}_{2}$ passivation on the detector surface. The other prominent feature in Fig. 5 is the typical X-ray absorbtion fine structure (XAFS) behaviour around the silicon $\mathrm{K}$ edge at $1.838 \mathrm{keV}$, enlarged in the inset of Fig. 5. At higher energies the solid line nicely fits the photon absorption data for $300 \mu \mathrm{m}$ of silicon. The solid line is a fit to the measured data with a depletion thickness of $298 \mu \mathrm{m}$. The QE is not supposed to change during the XMM lifetime under nominal conditions.

\subsection{Energy resolution}

The energy resolution is mainly determined by the statistical fluctuations of the ionization process (Fano noise), the charge transfer properties of the CCD and the electronic noise of the readout node. Figure 6 shows about 31 hours of in-orbit data with the internal calibration source in the "closed-cal" position from june 2000. The signals selected are only those which hit the CCD in the last 20 lines of the 12 CCDs, the area, which also contains the focal point. X-ray events from this region have undergone the 


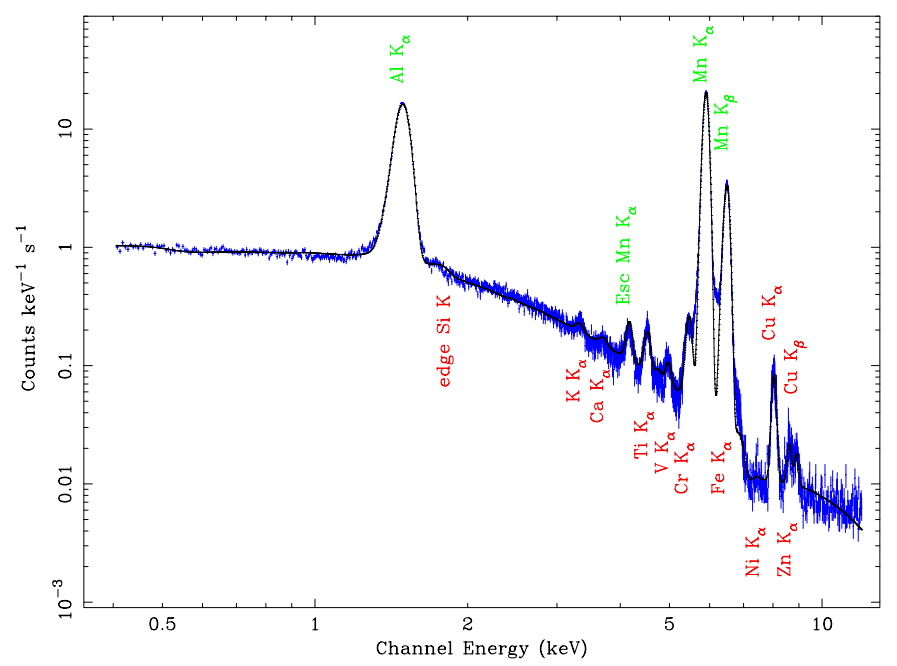

Fig. 6. Calibration spectrum with the internal radioactive source including the background with the filter wheel in closed position. The continuous background below the Mn lines arises mainly from photoelectrons stimulated from the ${ }^{55} \mathrm{Fe}$ source in the $\mathrm{Al}$ target. The iron $\mathrm{K}_{\alpha}$ line between $\mathrm{MnK}_{\alpha}$ and $\mathrm{MnK}_{\beta}$ is not resolved

maximum number of charge transfers and therefore the highest charge losses. The $\mathrm{AlK}_{\alpha}$, the $\mathrm{MnK}_{\alpha}$ and $\mathrm{MnK}_{\beta}$ and the $\mathrm{MnK}_{\alpha}$ escape peak are clearly visible. The $\mathrm{CuK}_{\alpha}$ and $\mathrm{CuK}_{\beta}$ peaks are fluorescence lines from the printed circuit board, generated by ionizing particles traversing the whole pn-CCD camera. The other fluorescence lines (e.g. $\mathrm{KK}_{\alpha}, \mathrm{TiK}_{\alpha}, \mathrm{VK}_{\alpha}, \mathrm{CrK}_{\alpha}, \mathrm{FeK}_{\alpha}, \mathrm{NiK}_{\alpha}, \mathrm{ZnK}_{\alpha}$ ) and others are trace elements in the aluminum structure of the camera and the invar ring holding the pn-CCD wafer. The energy resolution in the full frame mode is extracted from the internal calibration source including all kind of X-ray background. Over the first 9 months the peak position and the FWHM are shown in Figs. 7 and 8. At $\mathrm{MnK}_{\alpha}$ the FWHM is $161 \mathrm{eV}$ in the focal point, it is $152 \mathrm{eV}$ averaged over the whole CCD and is $140 \mathrm{eV}$ close to the readout nodes. The energy resolution improves in the extended full frame mode to $148 \mathrm{eV}$ (FWHM) averaged over the entire chip. The $\mathrm{AlK}_{\alpha}$ resolution is $111 \mathrm{eV}$ (FWHM) for the full frame and $105 \mathrm{eV}$ in the extended full frame mode. Due to the heavy overlap of many lines and because of the underlying continuous background the energy resolution is slightly better for monochromatic radiation.

\subsection{Instrument stability}

The long term instrument stability is checked routinely in terms of housekeeping data from all relevant camera parameters and by analysing the spectroscopic performance of the on-board calibration source. Figure 7 shows the variation of the $\mathrm{MnK}_{\alpha}$ peak position as a function of time after launch. Within less than $1 \mathrm{ADU}$ count $(5 \mathrm{eV})$ all measurment points are compatible with the pre-launch data.

The strong solar flare on July-14 did not leave any measurable damage in the pn-CCD camera.
The variation of the peak position (see Fig. 7), e.g. at the day 200 and 220 in the year 2000, was due to a strong temperature drop (approximately $10 \mathrm{~K}$ ) of electronic boxes outside of the camera housing, causing gain changes and shifts in the analog-to-digital conversion. The peak shift is correlated with satellite commands influencing the thermal budget. On ground, the change of the peak position is corrected. As can be seen in Fig. 8 the peak shift had no effect on the energy resolution. The error bars in Fig. 8 comprise only the statistical errors, systematics are not included. The larger error bars in CCD 10, 11 and 12 reflect the fact, that this area of the focal plane was only poorly illuminated by the calibration source. The variance of the FWHM at the MnK line including 36 extended calibration measurements over the last 9 months is only $1.8 \mathrm{eV}$. A peak shift due to CTE changes because of possible radiation damage is less than $5 \mathrm{eV}$.

\subsection{Instrument background}

As can be seen in Fig. 6 two other features of the spectrum need some explanation. (a) The continuous background from the lowest energies up to the MnK lines. This background is due to photo electrons from the $\mathrm{Al}$ fluorescence target, excited from the MnK X-rays from the calibration source. Because of the very thin radiation entrance window of the pn-CCD the low energy electrons can be clearly detected with high QE. This property is equally responsible for the high QE response for soft X-rays. (b) The flat background distribution for the highest energies arises from Compton electrons, generated by $\mathrm{X}$ - and gamma rays.

Another source of instrument background is caused by highly ionizing particles, being imaged by means of grazing incidence reflection through the X-ray telescope. They can be light and heavy ions as well as highly ionizing low energy protons (Aschenbach 2001). The duration of the flares can be of the order of minutes up to hours, their occurence is unpredictable (see e.g. Briel 2000). In case the detectors register a significant increase of counts, the filter wheel is put in closed position, but the EPIC instruments remain operational ${ }^{2}$.

The pn-CCD camera has the option to lower the gain of the signal processing electronics by a factor of 20 to increase the dynamic range in the so-called low-gain mode above $300 \mathrm{keV}$. This mode is very useful to study background phenomena. Figure 9 shows an example of such a measurement. During an observation in low-gain a sudden increase in count rate by a factor of 2.5 occurred in the CCD cameras without getting notice from the

\footnotetext{
${ }^{2}$ Observation is of course not possible, instrument data are not processed, but the detectors are still running. Extended instrument recovery times are avoided.
} 


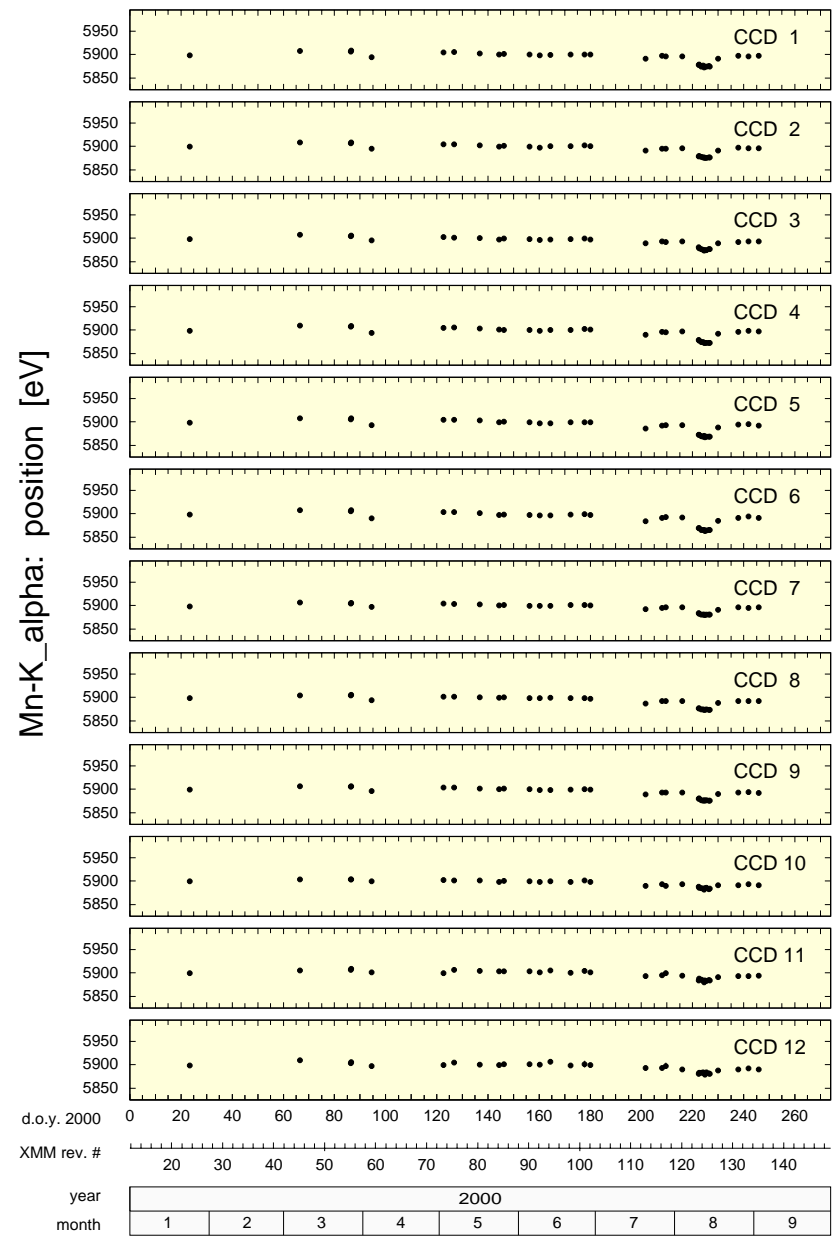

Fig. 7. Longterm stability of the pn-EPIC camera system monitored with the internal calibration source. Within a precision of $5 \mathrm{eV}$ no CTE related peak shifts were observed, given nominal operation temperatures in the pn-CCD camera system

radiation monitor ${ }^{3}$. The above threshold counter indicated an increased number of particles. The result of the analysis of those "soft proton flares" were summarized by Strüder et al. (2000):

1. The energy distribution of the protons has its maximum at the lowest measured energies at $1 \mathrm{keV}$ with an exponential attenuation of 4 orders of magnitude after $250 \mathrm{keV}$. In this measurement, $50 \mathrm{keV}$ of the proton's energy was already absorbed by the thick filter;

2. The protons show a clear vignetting proofing that they have been imaged through the telescope. We do not find the low energy protons in the $6 \mathrm{~cm}^{2}$ sensitive area outside the field of view. In contrast to minimum ionizing particles, the protons mainly produce single pixel events;

3. The protons lose typically $50 \mathrm{keV}$ of energy in the thick filters and about $20 \mathrm{keV}$ in the thin filters;

3 The low energy proton flare was still ongoing after the drop in count rate after $2.710^{4} \mathrm{~s}$, but the camera was automatically switched off and the filter wheel closed because of the high number of counts.

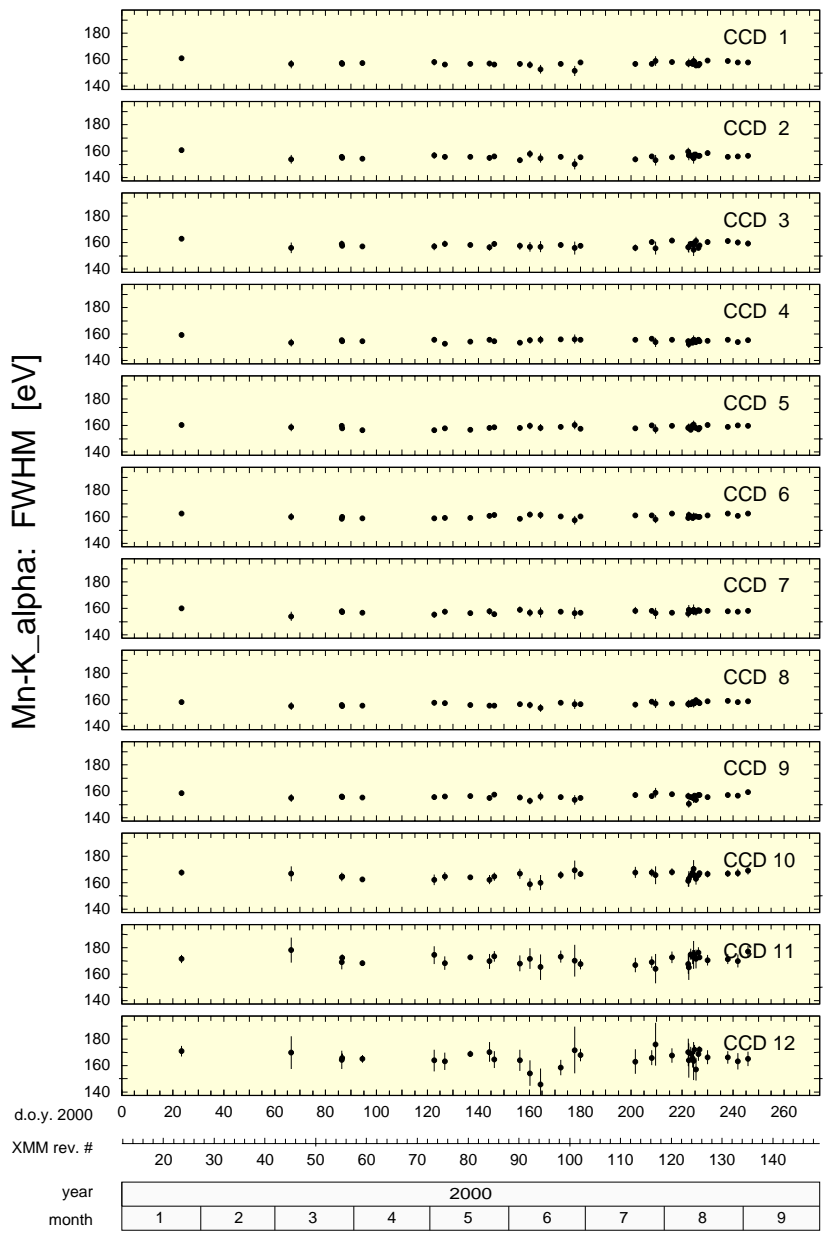

Fig. 8. The full width at half maximum of the internal calibration source. The larger errors in CCD's 10, 11 and 12 are due to the inhomogeneous focal plane illumination resulting in a reduced count rate

4. The soft proton flares heavily load the observational background (and the satellite telemetry) and therefore disturb and limit the observations, but they do not damage the instrument.

The lower (red) curve in Fig. 9 exactly represents the simulated spectrum of isotropically distributed mips crossing the pn-CCD's pixel structure. The difference of the lower and upper spectrum yields the low energy proton spectrum.

Minimum ionizing particles (mips) traverse the detector and leave about 80 electron-hole pairs per $\mu \mathrm{m}$ track length in the silicon. In a Monte-Carlo simulation we assumed a $4 \pi$ isotropic distribution of the mips, which nicely fits the measured data: The average energy deposition in one pixel is in the order of $50 \mathrm{keV}$ and the average number of pixels involved in a mip track is about 10 (Strüder 2000). The most probable track length in one single pixel is $150 \mu \mathrm{m}$. The onboard processor is able to remove almost $100 \%$ of the mips. The processing power onboard is not sufficient to remove them all. Post-processing on ground then rejects $100 \%$ of the mips. 


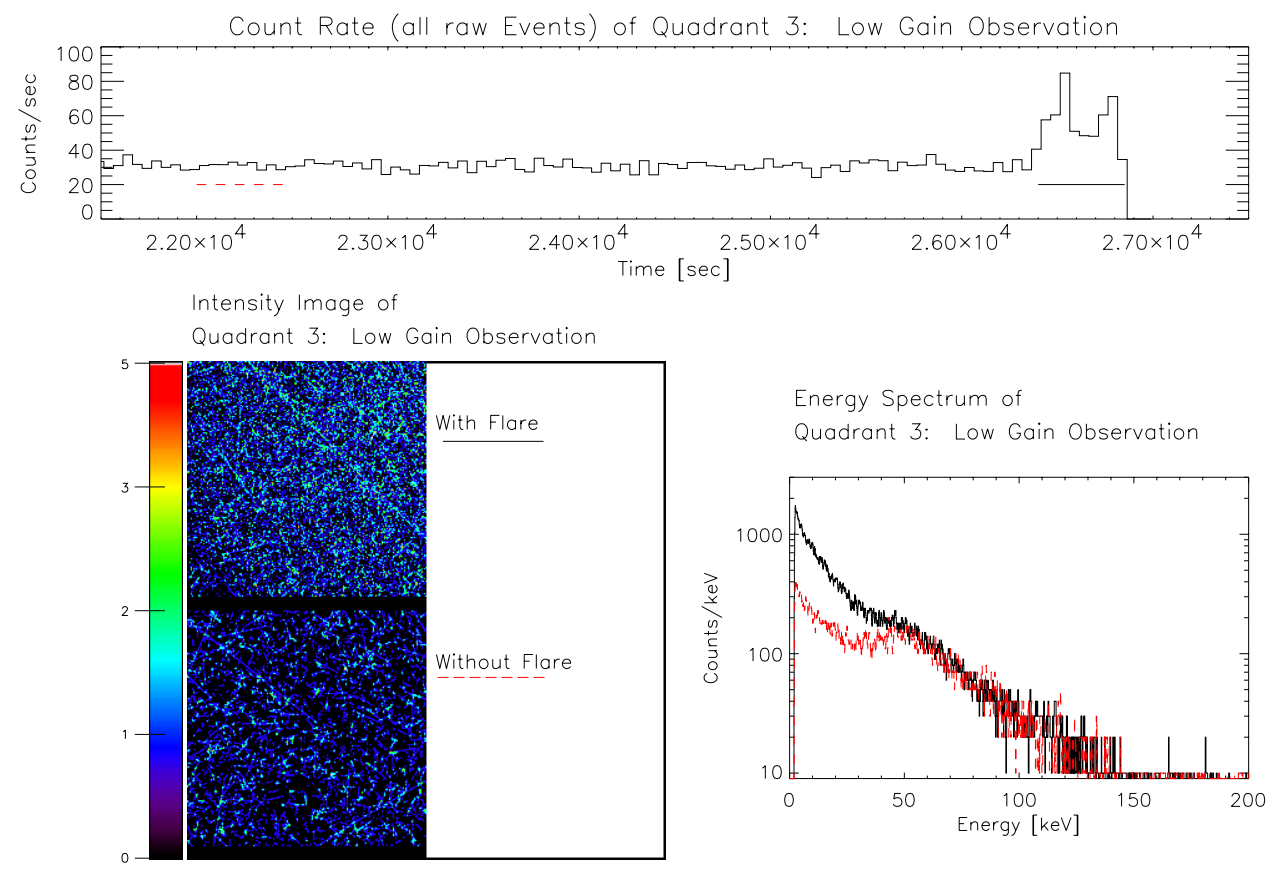

Fig. 9. Measured background spectra (thick filter) with a reduced gain (factor 20) during normal background, mainly mips (red line) and during a "soft proton flare" (black line). The upper panel shows the "light curve" of the observation with an increased background in the last $500 \mathrm{~s}$. The lower left picture shows the event pattern of the high and low background measurements. The lower right diagram shows the difference in energy deposition for the low and high background events

The instrument background was determined by measurements with the filter wheel closed. In the energy band of $2 \mathrm{keV}$ to $10 \mathrm{keV} 4.510^{-4}$ single events per sec, per keV and per $\operatorname{arcmin}^{2}$ were measured. For a circle with a radius of $7 \operatorname{arcsec}-$ i.e. the half energy width of the telescope system - this reduces to $1.510^{-4}$ per sec. The cosmic X-ray background within the 7 arcsec radius is $2.310^{-5}$ singles per sec. A circle with a radius of 7 arcsec characterizes the half energy width of the telescope and therefore gives a background estimate for a point source.

The analysis of the radiation background of all kinds will be an ongoing activity because of the lack of precise predictability, time transients and missing knowledge about the composition, energy profile, spatial distribution in the XMM orbit.

\section{Conclusions}

The EPIC pn-CCD camera on XMM-Newton from the Max-Planck-Institut für extraterrestrische Physik operates in orbit as calibrated on ground: The operating modes have been tested and qualified in-orbit and offer adequate readout schemes for all astronomical observations. The energy resolution is the same as in the ground calibrations and the instrument performance is stable with time, showing no radiation damage within the first nine months in orbit. The instrument background is in the process of being understood. Low energy protons can limit the sensitivity of observations, but do not damage the focal plane detector.
Acknowledgements. The XMM-Newton project is supported by the Bundesministerium für Bildung und Forschung/Deutsches Zentrum für Luft- und Raumfahrt (BMBF/DLR), the Max-Planck Society and the HeidenhainStiftung. In addition we would like to thank Kayser-Threde $\mathrm{GmbH}$ for the design of the pn camera head.

We are indebted to the staff of the MPI semiconductor laboratory, and the technical divisions at the MPE and IAAT.

The European Photon Imaging Camera was developed by the EPIC Consortium led by the Principal Investigator, Dr. M. J. L. Turner. The consortium comprises the following Institutes: MPE Garching, IAAT Tübingen, (Germany); University of Leicester, University of Birmingham, (UK); CEA/Saclay, IAS Orsay, CESR Toulouse, (France); IFC Milan, ITESRE Bologna, IAUP Palermo, Italy. EPIC is funded by: PPARC, CNES, DLR and ASI.

\section{References}

Aschenbach, B. 2001, to be published in NIM A

Briel, U., Aschenbach, B., Balasini, M., et al. 2000, SPIE, 4012, 154-164

Gatti, E., \& Rehak, P. 1984, NIM A, 225, 608-621

Hartmann, R., Strüder, L., Kemmer, J., et al. 1997, NIM A, 387, 241-248

Kendziorra, E., Clauß, T., Meidinger, N., et al. 2000, The effect of low energy protons on the performance of the EPIC pnCCD detector on XMM-Newton, to be published in SPIE, 4140

Kuster, M., Benlloch, S., Kendziorra, E., \& Briel, U. 1999, SPIE, 3765, 673-682

Meidinger, N., Schmalhofer, B., \& Strüder, L. 2000, NIM A, 439, 319-337 
Pfeffermann, E., Meidinger, N., Bräuninger, H., et al. 1999, SPIE, 3765, 184-191

Pinotti, E., Bräuninger, H., Findeis, N., et al. 1993, 326, 85-92 Soltau, H., Kemmer, J., Meidinger, N., et al. 2000, NIM A, 439, 547-559

Strüder, L., Holl, P., Lutz, G., \& Kemmer, J. 1987, NIM A, 253, 386-392

Strüder, L., Bräuninger, H., Meier, M., et al. 1990, NIM A,
$288,227-235$

Strüder, L., Bräuninger, H., Hartmann, R., et al. 1997, Rev. Sci. Ins. 68, 11, 4271-4274

Strüder, L., Meidinger, N., Pfeffermann, E., et al. 2000, SPIE, 4012, 342-352

Turner, M. J. L., Abbey, A., Arnaud, M., et al. 2001, A\&A, $365, \mathrm{~L} 27$ 